\title{
General Differential Galois Theory
} D. V. Trushin

\author{
Moscow State University, Faculty of Mechanics and Mathematics,
} Leninskie Gory, Moscow, 119899, Russia

Received September 2, 2009

\begin{abstract}
The general Galois theory of arbitrary nonlinear partial differential equations is presented in the paper. For each system of differential equations its splitting field and the differential Galois group are defined. The main result is the theorem on the Galois correspondence for normal extensions.
\end{abstract}

DOI: $10.3103 / \mathrm{S} 0027132210030071$

At present time, there are many different kinds of differential Galois theories. A detailed review of such theories was given in [1, pp. 135-137]. However, most general results were obtained by methods of the model theory $[2$, Ch. $6, \S 6.2$ and Ch. $18, \S 18.3]$. In this paper we present the Galois theory of arbitrary systems of partial differential equations. Its distinguishing feature is the application of a purely algebraic technique without using the model theory.

In order to meet the needs of our theory, it is required to develop the corresponding differential-algebraic technique whose heart is the following theorem.

Theorem 1. Let $K$ be a differential field and $B_{\alpha}$ be a family of differentially finitely generated algebras over $K$. Then there exists a prime differential ideal $\mathfrak{p}$ in the ring $\otimes_{\alpha} B_{\alpha}$ possessing one of the following properties:

1) the constants of the residue field of the ideal $\mathfrak{p}$ are algebraic over the constants of the field $K$;

2) any differentially finitely generated subalgebra in the residue field of the ideal $\mathfrak{p}$ is locally simple;

3) any homomorphism of the field $K$ to a differentially closed field can be extended to a differential homomorphism of the residue field of the ideal $\mathfrak{p}$.

In the model theory there is a notion of constructible model [2, Ch. 10, § 10.4]. We give an algebraic analogue of this notion, which plays a key role in understanding the structure of differential fields.

We define the notion of a splitting field in the following way. Let $F_{\alpha}$ be a family of systems of differential equations. Construct the family $B_{\alpha}=K\left\{y_{1}, \ldots, y_{n_{\alpha}}\right\} /\left[F_{\alpha}\right]$. We say that $L$ is a splitting field of the family $\left\{B_{\alpha}\right\}$ over $K$ if the following conditions are satisfied:

1 ) for any $\alpha$ and any locally maximal ideal $\mathfrak{m}$ in $L \otimes B_{\alpha}$ the equality $\left(L \otimes B_{\alpha}\right) / \mathfrak{m}=L$ holds $(L$ is sufficiently large);

2 ) the field $L$ is generated by the family $\left\{B_{\alpha}\right\}$ ( $L$ is not too large);

3 ) for any field $L^{\prime}$ satisfying conditions (1) and (2) there exists an embedding of $L$ into $L^{\prime}$ over $K$ ( $L$ is universal).

After developing the algebraic technique, we use it to show that for any family of systems of differential equations there exists a splitting field unique up to an isomorphism. Moreover, such a field is constructible over the basic field $K$.

The Galois correspondence for splitting fields can be described as follows. Given an arbitrary splitting field $L$ over $K$, consider the family $\mathcal{F}$ of subfields in $L$ which $L$ is constructible over. Denote the group of differential automorphisms of $L$ over $K$ by $G$. Introduce the set $\mathcal{G}=\left\{H \subseteq G \mid H=\mathrm{Gal}^{\Delta}\left(L / L^{H}\right), L^{H} \in \mathcal{F}\right\}$. Let $\mathcal{N}$ be the set of all splitting subfields in $L$. Then the following theorem on the correspondence is valid.

Theorem 2. In the above notations the following statements are valid:

1) the mappings $F \mapsto \operatorname{Gal}^{\Delta}(L / F)$ and $H \mapsto L^{H}$ are mutually inverse bijections between $\mathcal{F}$ and $\mathcal{G}$;

2) the described correspondence defines a bijection between the set $\mathcal{N}$ and the set of all normal subgroups in $\mathcal{G}$, and for any invariant subfield $F^{\prime}$ the equality $\mathrm{Gal}^{\Delta}\left(F^{\prime} / F\right)=\mathrm{Gal}^{\Delta}(L / F) / \mathrm{Gal}^{\Delta}\left(L / F^{\prime}\right)$ holds;

3) the cardinality of the set of extensions of an automorphism $\varphi: F \rightarrow F$ to an automorphism of $L$ does not depend on the automorphism $\varphi$.

\section{REFERENCES}

1. P. J. Cassidy and M. F. Singer, "Galois Theory of Parameterized Differential Equations and Linear Differential Algebraic Groups," in Differential Equations and Quantum Groups (EMS Publishing House, 2006), pp. $113-157$.

2. B. Poizat and M. Klein, A Course in Model Theory: an Introduction to Contemporary Mathematical Logic (Springer-Verlag, N.Y., 2000).

Translated by A. Oshemkov 